\title{
Can Non-Polio Enteroviruses Be Tamed with a Vaccine to Minimize Paralysis Caused by Them?
}

\author{
Omesh Kumar Bharti \\ Corporation Health Officer, Municipal Corporation, Shimla, India \\ Email: bhartiomesh@yahoo.com
}

Received 16 January 2015; accepted 30 January 2015; published 3 February 2015

Copyright (C) 2015 by author and Scientific Research Publishing Inc.

This work is licensed under the Creative Commons Attribution International License (CC BY). http://creativecommons.org/licenses/by/4.0/

\section{(c) (i) Open Access}

\section{Abstract}

Background: While we are inching towards global eradication of polio, the paralysis due to nonpolio viruses (NPEV) poses greater challenge. Factors responsible for causing Acute Flaccid Paralysis (AFP) were studied in 3596 AFP patients in 64 districts of Uttar-Pradesh, India, to observe indirect relationship of AFP with wild polio as well as NPEV. A recent study suggests the need to investigate polio virus negative but NPEV positive AFP cases. Methods: The lab results of the stool samples of these children were line listed and analysed to observe the association of various factors with respect to presence of paralysis on 60 follow-up days. Taking zero OPV dose AFP cases as a biological base, we studied the relationship of presence of paralysis at 60 follow-up days to that of presence of NPEV in stool samples while polio virus was present or absent. Results: 70 of the 86 AFP cases (81\%) with zero OPV dose and having only NPEV isolated in stool samples were having paralysis at 60 follow-up days. There were $4.54 \%$ (162) AFP cases, which did not carry any polio virus but were having NPEV isolated in the stool samples and paralysis at 60 follow-up days. $79 \%$ (75/95) of zero OPV dose children, who were having residual weakness at 60 follow-up days, were carrying both polio virus as well as NPEV in their stool samples. Total AFP cases, having residual weakness at 60 follow-up days and having NPEV in stool samples, decreased with increase in OPV doses; a behavior similar to what wild polio viruses (WPV) have to OPV. Conclusions: Maybe polio like NPEV is active for causing severe paralysis in children and is responding to the OPV. As is evident in the studies by M. Margalith, B. Fattal et al. [1] that there is an antibody response to the enteroviruses, we can think of coming out with a vaccine against the enteroviruses. Therefore, enterovirus vaccine can be produced on similar lines to that of OPV, as now we have enough isolates of NPEV. Effective NPEV surveillance system also needs to be in place.

\section{Keywords}

Polio, Non-Polio, Paralysis, Enteroviruses, Vaccine 


\section{Introduction}

While we are inching towards global eradication of polio, the paralysis due to non-polio enteroviruses (NPEV) poses greater challenge now. Polio eradication is the main agenda of the Global Polio Eradication Initiative (GPEI) and in India remarkable work has been done to become polio free. It is a fact that there is ever increase in AFP cases from 9461 in 1998 to 44,846 in 2008 (Table 1) pushing AFP rate to 10 times than expected (Figure 1). Despite the fact that there has been sharp decline in polio virus isolation in AFP cases over the years, the AFP rate in Uttar Pradesh touched 22.4 in 2008. So, the issue is what is causing high incidence of paralysis (AFP epidemic) when polio virus is on the verge of eradication? Now more children in India are getting paralysis than earlier, which demands immediate attention as the total AFP rate goes many folds up than expected. Even if polio is eradicated, the paralysis due to other viruses is replacing the burden of disability earlier caused by polio viruses as observed by James A Ayukekbong, Tomas Bergström et al. [2]. A vision of world free of paralysis will remain a distant dream if the paralysis continues due to non-polio viruses. The author, while working with polio eradication initiative, found a strange association between paralysis present at 60 follow-up days and presence of NPEV in the stool samples of the affected children. This led the author to investigate deeper into the cause. Laxmivandana R, Yergolkar $\mathrm{P}$ et al. suggest the need to investigate polio virus negative but NPEV positive AFP cases [3] and this study responds to this suggestion.

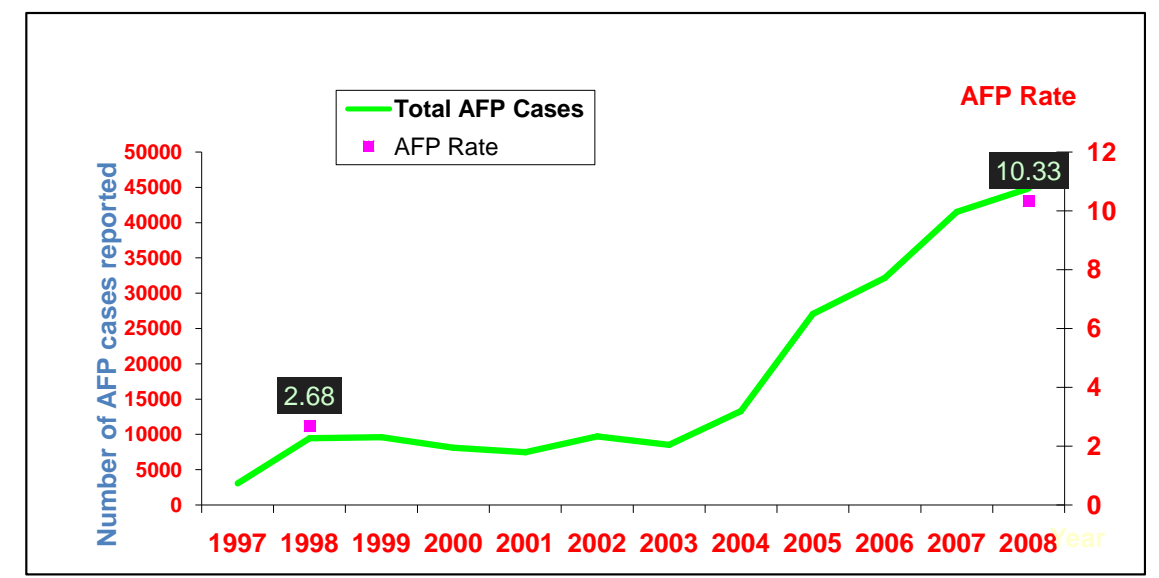

Figure 1. No. of AFP and AFP rate 1997-2008.

Table 1. Alarming rise in AFP cases, India.

\begin{tabular}{lcc}
\hline Year & AFP Cases \\
1997 & 3047 \\
1998 & 9461 \\
1999 & 9587 \\
2000 & 8103 \\
2001 & 7470 \\
2002 & 9705 \\
2003 & 8505 \\
2004 & 13,274 \\
2005 & 27,050 \\
2006 & 32,173 \\
2007 & 41,522 \\
2008 & 44,846 \\
\hline
\end{tabular}




\section{Methodology}

We did a secondary data analysis of AFP cases in high endemic districts of Uttar Pradesh, India. 3596 children suffering from AFP in 64 districts of Uttar Pradesh were included in the study during the period Jan 1998-July 1999. The lab results of the stool samples from these children were line listed and analysed to observe the association between various factors with respect to presence of paralysis on 60 day follow-up. The data was analysed using Microsoft excel and Epi-info software.

Taking zero OPV dose AFP cases as a biological base, we studied the relationship of presence of paralysis at 60 days follow-up to that of presence of NPEV in stool samples while polio virus was present or absent. We also studied the relationship of increase in OPV doses with that of presence of paralysis in AFP cases having Polio or NPEV viruses.

\section{Results}

The line-list of all the 3596 cases was studied and following observations were made:

1) 70 out of 86 AFP cases (81\%) with only NPEV in stool samples and with zero OPV dose were having residual weakness at 60 days follow-up.

2) $4.5 \%$ children (162) with paralysis at 60 days follow-up were though not carrying polio virus but were having NPEV isolated in their stool sample.

3) $79 \%$ (75/95) of zero OPV dose children, who were having residual weakness at 60 days follow-up, were carrying both Polio virus as well as NPEV in their stool samples. There appears to be a relation between paralysis caused by the poliovirus and presence of NPEV, making this paralysis more severe i.e. the paralysis was present in majority even after 60 days of follow-up where we were able to isolate both wild polio viruses (WPV) and NPEV in stool samples.

4) With increase in OPV doses, number of AFP cases with Residual Weakness at 60 days follow-up tend to decrease. This decrease was observed both in cases of those having either polio virus (Figure 2) or NPEV (Figure 3) in stool samples, showing a pattern of association similar as WPV responds to OPV.

5) In $10 \%$ of total cases (364), where there was no isolation of either Poliovirus or NPEV, the paralysis was present, and is not explainable due to the other factors of differential diagnosis of Polio and need further research.

\section{Discussion}

There is interaction between the Polio Virus and the Enteroviruses in the intestine of the children in India and this interaction explains the severity of the paralysis (79\%) in case both Polio virus and NPEV are isolated in the stool samples. Also, the severity (no. of cases with paralysis at 60 days follow-up) decreases with the increase of

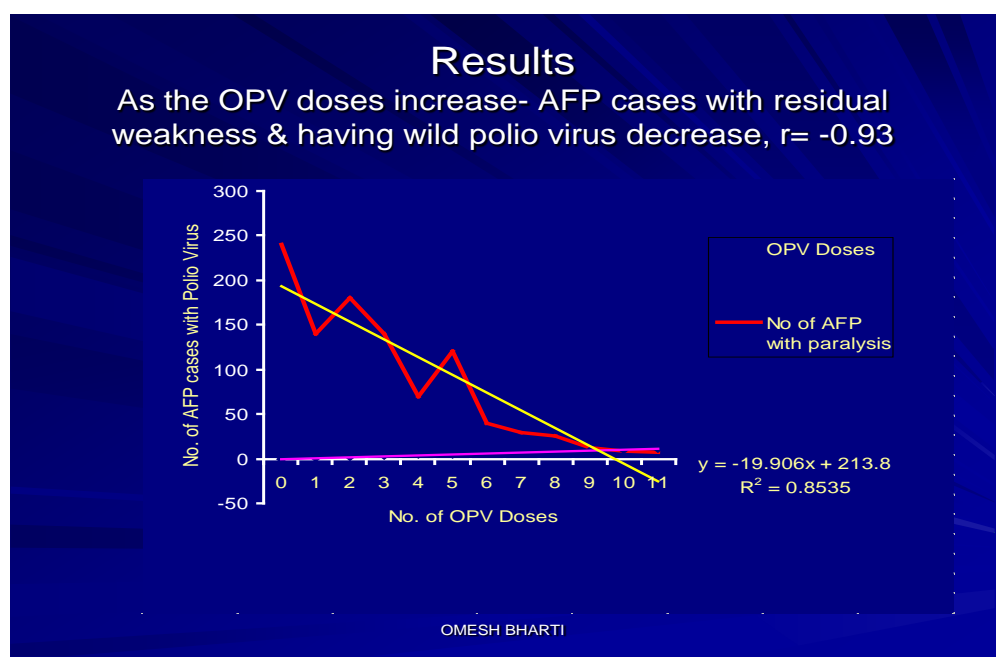

Figure 2. As the OPV doses increase-AFP cases with residual weakness but having wild polio virus decrease, $r=-0.93$. 


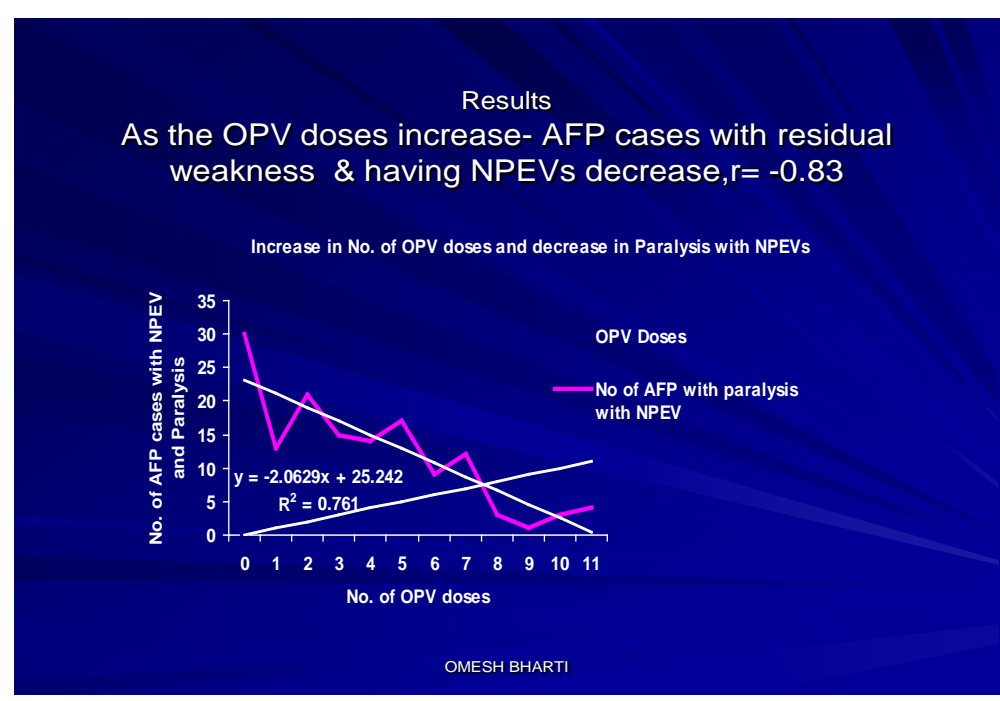

Figure 3. As the OPV doses increase-AFP cases with residual weakness but having NPEVs decrease, $r=-0.83$.

doses of OPV, is a pointer towards an association between these viruses.

In $4.5 \%$ AFP cases, there was isolation of the NPEV in stool samples without polio virus and associated paralysis. It needs further studies.

The increase in AFP cases after the decrease in Polio cases per se in India is also explainable due the facts given here and it can be assumed that in the light of the evidence of NPEV causing paralysis and the fact that the polio virus is no more there in the intestine to compete with the NPEV, the number of AFP cases is increasing.

There is low efficacy of trivalent OPV vaccine in India (11\% - 23\%) [4], this may be because of the fact that type $2(\mathrm{OPV})$ vaccine virus and enteric pathogens often interfere with responses to types 1 and 3 vaccine viruses as reported by Patriarca PA, Wright PF et al. [5]; the corollary holds true for OPV and NPEV interaction.

Deshpande JM, Kamat JR et al. [6] indicate that there is an antibody response to the enteroviruses, therefore, we can think of coming out with a vaccine against the enteroviruses where we suspect NPEV to be the cause of paralysis. It would be easy to do this at the moment, when we have all the infrastructure and technology to produce OPV Vaccine. The enterovirus vaccine can be produced on similar lines, as now we have a database of all the enterovirus strains in the country [7]. Moreover, the number of cases with residual paralysis at the time of initiation of the AFP surveillance was less than they are now, so more and more children are getting crippled now than before and we have a moral obligation to investigate the causes and give appropriate solutions.

In 1975 epidemic of CNS Disease that occurred in Bulgaria, all the isolates belonged to single antigenic type, related to American and Swedish strain of enterovirus 71 and antibody to enterovirus 71 was found in $72 \%$ of 392 patients with a history of poliomyelitis like disease that year. In a large mixed epidemic of CNS Disease in Hungary in 1978 with 724 cases of Encephalitis and 45 deaths, enterovirus 71 was isolated in polio like cases. The poliovirus infection was excluded by neutralization tests. In US in the year 1987, 53\% of (24 of 45) patients infected with enterovirus 71 had CNS involvement with 6 developing paralysis. All these reports as mentioned in Viral Infections of Humans; Fourth edition [8], clearly point towards greater role enteroviruses may be playing in causing polio like paralytic disease in India as is evident due to very high AFP rate.

Enterovirus 71 has caused large outbreaks of HFMD worldwide, especially in children in Asia. Some infections from this virus have been associated with severe neurologic disease, such as brainstem encephalitis [9]. Effort to produce a vaccine against NPEV [10] are welcome and need further prioritisation.

\section{Conclusion}

A polio like enterovirus is active in causing paralysis and is responding to OPV in a similar fashion. As our data is based on minimum of $10 \%$ of isolation of NPEV in stool samples as sensitivity criteria of polio eradication campaign, the actual paralysis (AFP) caused by NPEV must be higher at 60 follow-up days. 


\section{Recommendation}

This being a pilot study, more such studies can throw light on the direct relationship of NPEV causing paralysis in AFP cases. The response of NPEV to OPV also needs further studies. Since NPEVs are now known to cause paralysis [11] and also known to generate antibody response in the human body, may be a vaccine to tame them would help children get rid of persistent paralysis despite polio nowhere in the picture. The role of NPEV as causative agent of paralysis in countries that have eradicated polio, especially tropical one's, needs to be understood in the context of paralysis present in less than 15 years of age despite zero polio status. A non-polio surveillance system needs to be in place in India along with environmental surveillance of polio/non-polio viruses to recognize non-polio paralysis and associated outbreaks that we may be missing at the moment in the absence of such a surveillance system.

\section{Limitations}

1) Serological evidence to show NPEV antibodies in the serum of the affected children could not be done; we could only generate indirect evidence to show the co-relation between polio virus and NPEV.

2) There may be cases of AFP that were because of vaccine associated paralysis (VAP) or due to vaccine derived polio virus (VDPV) and neither due to polio nor due to NPEV, and need further studies.

3) In the absence of Lab support we could not type different NPEVs to identify the causative one.

4) In the absence of availability of the latest AFP Lab data we could not assess the current status of AFP with respect to NPEVs.

5) This study could not through light on other causes of the AFP paralysis that may be there apart from NPEV.

\section{Acknowledgements}

Our sincere thanks to National Polio Surveillance Project, India, Government of Himachal Pradesh, India, National Foundation For Infectious Diseases, Bathesda, USA, Dr. Bruce G. Weniger, MD, formally with National Center for Immunization \& Respiratory Diseases, CDC, GA and Ms. Padmini Menon, Moderator e-group www.technet21.org.

\section{References}

[1] Margalith, M., Fattal, B., Shuval, H.I., Morag, A., et al. (1986) Prevalence of Antibodies to Enteroviruses and Varicella-Zoster Virus among Residents and Overseas Volunteers at Agricultural Settlements in Israel. Journal of Medical Virology, 20, 189-197. http://onlinelibrary.wiley.com/doi/10.1002/jmv.1890200211/abstract

[2] Ayukekbong, J.A. and Bergström, T. (2014) Polio Will Go, Acute Flaccid Paralysis Will Stay. Vol. $383,2209$. www.thelancet.com http://www.thelancet.com/pdfs/journals/lancet/PIIS0140-6736\%2814\%2961080-1.pdf

[3] Laxmivandana, R., Yergolkar, P., Gopalkrishna, V. and Chitambar, S.D. (2013) Characterization of the Non-Polio Enterovirus Infections Associated with Acute Flaccid Paralysis in South-Western India. PLoS ONE, 8, e61650. http://dx.doi.org/10.1371/journal.pone.0061650

[4] Grassly, N.C., Fraser, C., Wenger, J., Deshpande, J.M., Sutter, R.W., Heymann, D.L. and Aylward, R.B. (2006) New Strategies for the Elimination of Polio from India. Science, 314, 1150-1153. http://www.imedicinas.com/pfw_files/cma/gdo_upload/m503/PolioOral.pdf

[5] Patriarca, P.A., Wright, P.F. and John, T.J. (1991) Factors Affecting the Immunogenicity of Oral Poliovirus Vaccine in Developing Countries: Review. Reviews of Infectious Disease, 13, 926-939. http://www.ncbi.nlm.nih.gov/pubmed/1660184

[6] Deshpande, J.M., Kamat, J.R., Rao, V.K., Nadkarni, S.S., Kher, A.S., Salgaokar, S.D. and Rodrigues, J.J. (1995) Prevalence of Antibodies to Polioviruses \& Enteroviruses Excreted by Healthy Children in Bombay. Indian Journal of Medical Research, 101, 50-54. http://www.ncbi.nlm.nih.gov/pubmed/7729847

[7] Dhole, T.N., Ayyagari, A., et al. (2009) Non-Polio Enteroviruses in Acute Flaccid Paralysis Children of India: Vital Assessment before Polio Eradication. Journal of Paediatrics and Child Health, 45, 409-413. http://dx.doi.org/10.1111/j.1440-1754.2009.01529.x http://www.ncbi.nlm.nih.gov/pmc/articles/PMC3632520/

[8] Melnick, J.L. (1997) Poliovirus and Other Enteroviruses. In: Evans A.S. and Kaslow, E.A., Eds., Viral Infection of 
Humans, Epidemiology and Control, Fourth Edition, Plenum Medical Book Company, New York and London, 583648. http://www.amazon.com/Viral-Infections-Humans-Epidemiology-Control/dp/0306448564

[9] MedicineNet.com: http://www.medicinenet.com/script/main/hp.asp

[10] Mat-Rahim, N.-A. and AbuBakar, S. (2014) Human Enterovirus 71 DNA Vaccine Constructs Containing 5’UTR with Complete Internal Ribosome Entry Site Sequence Stimulated Improved Anti-Human Enterovirus 71 Neutralizing Immune Responses. World Journal of Vaccines, 4, 33-43.

http://www.scirp.org/journal/PaperInformation.aspx?paperID=43089

[11] Saeed, M., Zaidi, S., Naeem, A., Masroor, M., Sharif, S., Shaukat, S., Angez, M. and Khan, A. (2007) Epidemiology and Clinical Findings Associated with Enteroviral Acute Flaccid Paralysis in Pakistan. BMC Infectious Diseases, 7, 6. http://www.biomedcentral.com/1471-2334/7/6 http://dx.doi.org/10.1186/1471-2334-7-6 
Scientific Research Publishing (SCIRP) is one of the largest Open Access journal publishers. It is currently publishing more than 200 open access, online, peer-reviewed journals covering a wide range of academic disciplines. SCIRP serves the worldwide academic communities and contributes to the progress and application of science with its publication.

Other selected journals from SCIRP are listed as below. Submit your manuscript to us via either submit@scirp.org or Online Submission Portal.
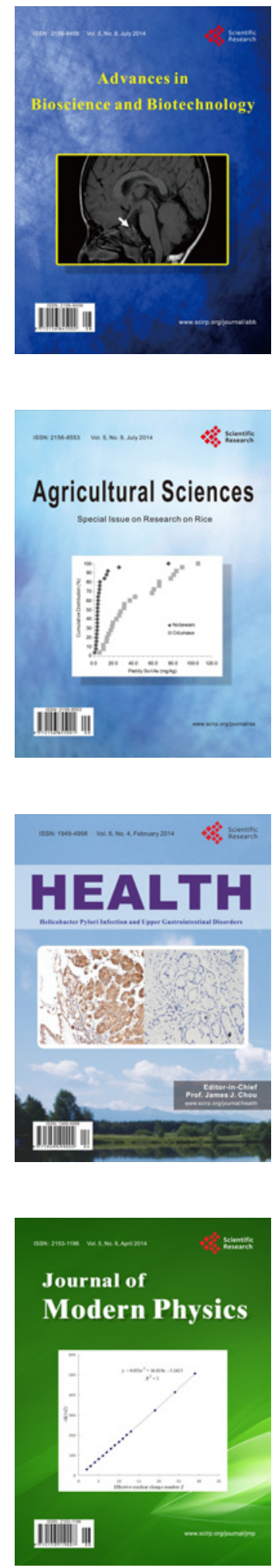
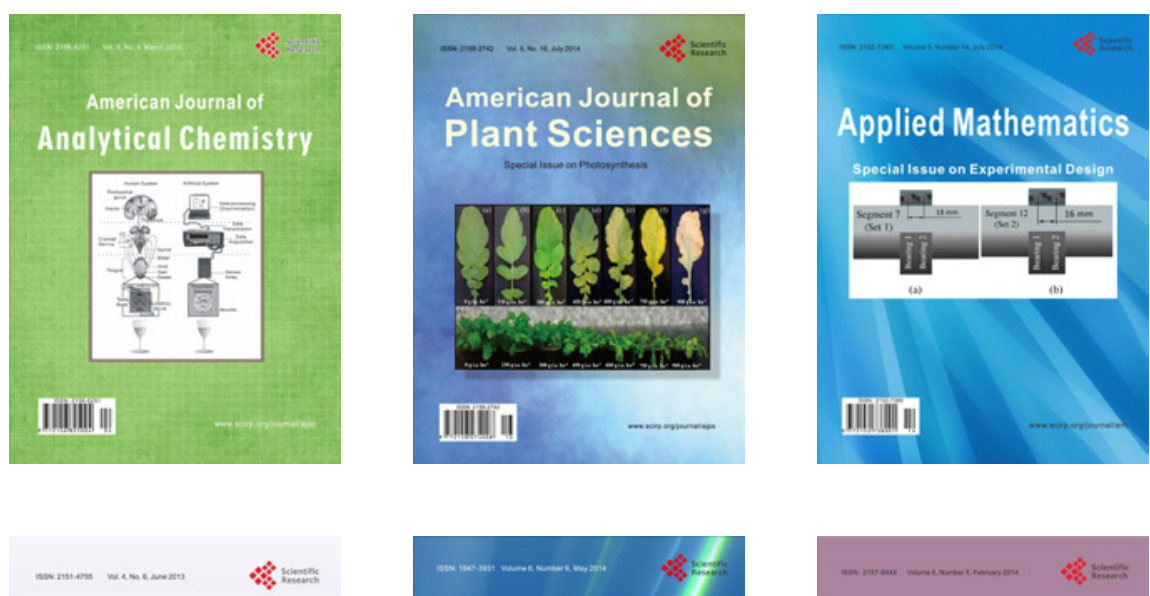

Creative Education
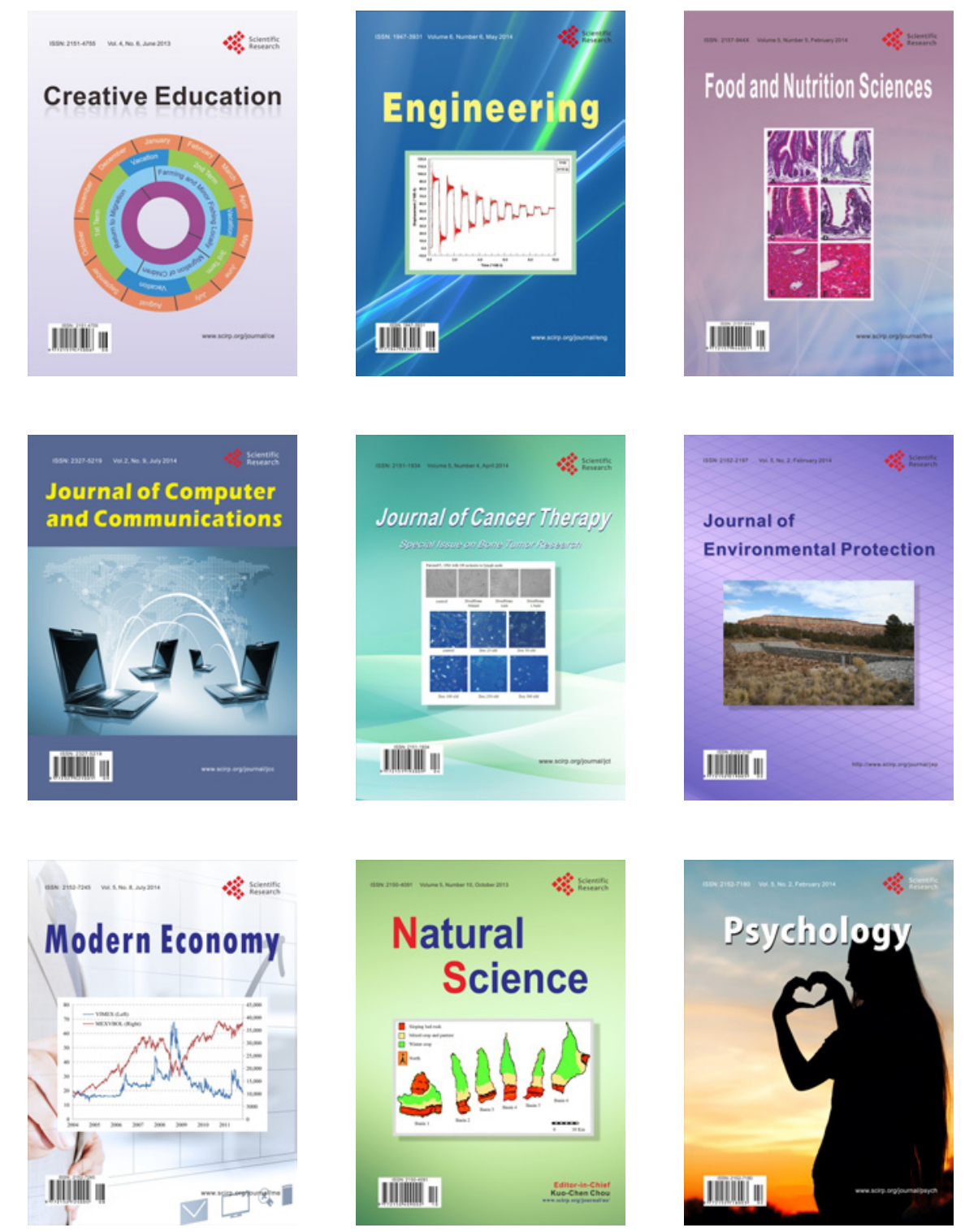